PROCEEDINGS OF THE

AMERICAN MATHEMATICAL SOCIETY

Volume 139, Number 7, July 2011, Pages 2357-2366

S 0002-9939(2010)10685-8

Article electronically published on December 8, 2010

\title{
SYMBOLIC POWERS AND MATROIDS
}

\author{
MATTEO VARBARO
}

(Communicated by Irena Peeva)

\begin{abstract}
We prove that all the symbolic powers of a Stanley-Reisner ideal
\end{abstract} $I_{\Delta}$ are Cohen-Macaulay if and only if the simplicial complex $\Delta$ is a matroid.

\section{INTRODUCTION}

Stanley-Reisner rings supply a bridge between combinatorics and commutative algebra, attaching to any simplicial complex $\Delta$ on $n$ vertices the Stanley-Reisner ideal $I_{\Delta}$ and the Stanley-Reisner $\operatorname{ring} K[\Delta]=S / I_{\Delta}$, where $S$ is the polynomial ring on $n$ variables over a field $K$. One of the most interesting parts of this theory is finding relationships between combinatorial and topological properties of $\Delta$ and ring-theoretic properties of $K[\Delta]$. For instance, it is a wide open problem to characterize graph-theoretically the graphs $G$ for which $K[\Delta(G)]$ is Cohen-Macaulay, where $\Delta(G)$ denotes the independence complex of $G$. In [TY, Theorem 3], Terai and Yoshida proved that $S / I_{\Delta}^{m}$ is Cohen-Macaulay for any $m \in \mathbb{N}_{\geq 1}$ if and only if $I_{\Delta}$ is a complete intersection. Because it is a general fact that all the powers of any homogeneous complete intersection ideal are Cohen-Macaulay, somehow the above result says that there are no Stanley-Reisner ideals with this property except for the trivial ones. Since if $S / I_{\Delta}^{m}$ is Cohen-Macaulay, then $I_{\Delta}^{m}$ is equal to the $m$ th symbolic power $I_{\Delta}^{(m)}$ of $I_{\Delta}$, it is natural to ask:

For which $\Delta$ is the ring $S / I_{\Delta}^{(m)}$ Cohen-Macaulay for any $m \in \mathbb{N}_{\geq 1}$ ?

The answer is amazing. In this paper we prove that $S / I_{\Delta}^{(m)}$ is Cohen-Macaulay for any $m \in \mathbb{N}_{\geq 1}$ if and only if $\Delta$ is a matroid (Theorem 2.1). The above result is proved independently and with different methods by Minh and Trung in [MT, Theorem 3.5]. Matroid is a well-studied concept in combinatorics, and it was originally introduced as an abstraction of the notion of the set of bases of a vector space. The approach to prove the above result is not direct, passing through the study of some blowup algebras related to $\Delta$. Among the consequences of Theorem 2.1 we remark Corollary 2.9. After localizing at the maximal irrelevant ideal, $I_{\Delta}$ is a set-theoretic complete intersection whenever $\Delta$ is a matroid.

Received by the editors March 14, 2010 and, in revised form, June 25, 2010.

2010 Mathematics Subject Classification. Primary 13A15, 05E45; Secondary 13A30. 


\section{The RESUlT}

In this section we prove the main theorem of the paper.

2.1. Definition of the basic objects. First of all we define the basic objects involved in the statement. For the part concerning commutative algebra and StanleyReisner rings, we refer to Bruns and Herzog $\mathrm{BH}$, Stanley [St, or Miller and Sturmfels MS. For what concerns the theory of matroids, some references are the book of Welsh We or that of Oxley $\mathrm{Ox}$.

Let $K$ be a field, $n$ a positive integer and $S=K\left[x_{1}, \ldots, x_{n}\right]$ the polynomial ring on $n$ variables over $K$. Also, $\mathfrak{m}$ is the maximal irrelevant ideal of $S$. We denote the set $\{1, \ldots, n\}$ by $[n]$. By a simplicial complex $\Delta$ on $[n]$ we mean a collection of subsets of $[n]$ such that for any $F \in \Delta$, if $G \subseteq F$, then $G \in \Delta$. An element $F \in \Delta$ is called a face of $\Delta$. The dimension of a face $F$ is $\operatorname{dim} F=|F|-1$, and the dimension of $\Delta$ is $\operatorname{dim} \Delta=\max \{\operatorname{dim} F: F \in \Delta\}$. The faces of $\Delta$ which are maximal under inclusion are called facets. We denote the set of the facets of $\Delta$ by $\mathcal{F}(\Delta)$. For a simplicial complex $\Delta$ we can consider a square-free monomial ideal, known as the Stanley-Reisner ideal of $\Delta$ :

$$
I_{\Delta}=\left(x_{i_{1}} \cdots x_{i_{s}}:\left\{i_{1}, \ldots, i_{s}\right\} \notin \Delta\right) .
$$

The $K$-algebra $K[\Delta]=S / I_{\Delta}$ is called the Stanley-Reisner ring of $\Delta$, and it turns out that

$$
\operatorname{dim}(K[\Delta])=\operatorname{dim} \Delta+1
$$

More precisely, with the convention of denoting by $\wp_{A}=\left(x_{i}: i \in A\right)$ the prime ideal of $S$ generated by the variables associated to a given subset $A \subseteq[n]$, we have

$$
I_{\Delta}=\bigcap_{F \in \mathcal{F}(\Delta)} \wp[n] \backslash F .
$$

Given any ideal $I \subseteq S$ its $m$ th symbolic power is $I^{(m)}=\left(I^{m} S_{W}\right) \cap S$, where $W$ is the complement in $S$ of the union of the associated primes of $I$ and $S_{W}$ denotes the localization of $S$ at the multiplicative system $W$. If $I$ is a square-free monomial ideal, then $I^{(m)}$ is just the intersection of the (ordinary) powers of the minimal prime ideals of $I$. Thus

$$
I_{\Delta}^{(m)}=\bigcap_{F \in \mathcal{F}(\Delta)} \wp_{[n] \backslash F}^{m} .
$$

The last concept which is needed to understand the main theorem of the paper is a matroid. A simplicial complex $\Delta$ on $[n]$ is said to be a matroid if, for any two facets $F$ and $G$ of $\Delta$ and any $i \in F$, there exists a $j \in G$ such that $(F \backslash\{i\}) \cup\{j\}$ is a facet of $\Delta$. It is well known that if $\Delta$ is a matroid, then $K[\Delta]$ is Cohen-Macaulay. In particular, all the facets of a matroid have the same dimension. A useful property of matroids is the following.

Exchange property. Let $\Delta$ be a matroid on $[n]$. For any two facets $F$ and $G$ of $\Delta$ and for any $i \in F$, there exists $j \in G$ such that both $(F \backslash\{i\}) \cup\{j\}$ and $(G \backslash\{j\}) \cup\{i\}$ are facets of $\Delta$.

2.2. Statement and proof. What we are going to prove is the following theorem.

Theorem 2.1. Let $\Delta$ be a simplicial complex on $[n]$. Then $S / I_{\Delta}^{(m)}$ is CohenMacaulay for any $m \in \mathbb{N}_{\geq 1}$ if and only if $\Delta$ is a matroid.

Remark 2.2. Notice that Theorem 2.1 does not depend on the characteristic of $K$. 
Remark 2.3. If $\Delta$ is the $k$-skeleton of the $(n-1)$-simplex, $-1 \leq k \leq n-1$, then $\Delta$ is a matroid. So Theorem 2.1 implies that all the symbolic powers of $I_{\Delta}$ are Cohen-Macaulay.

In order to prove Theorem 2.1] it is useful to introduce another square-free monomial ideal associated to a simplicial complex $\Delta$, namely the cover ideal of $\Delta$ :

$$
J(\Delta)=\bigcap_{F \in \mathcal{F}(\Delta)} \wp_{F} .
$$

We have $\operatorname{dim}(S / J(\Delta))=n-\operatorname{dim} \Delta-1$. The name "cover ideal" comes from the following fact: A subset $A \subseteq[n]$ is called a vertex cover of $\Delta$ if $A \cap F \neq \emptyset$ for any $F \in \mathcal{F}(\Delta)$. Then it is easy to see that

$$
J(\Delta)=\left(x_{i_{1}} \cdots x_{i_{s}}:\left\{i_{1}, \ldots, i_{s}\right\} \text { is a vertex cover of } \Delta\right) .
$$

Let $\Delta^{c}$ be the simplicial complex on $[n]$ whose facets are $[n] \backslash F$ such that $F \in \mathcal{F}(\Delta)$. Clearly we have $I_{\Delta^{c}}=J(\Delta)$ and $I_{\Delta}=J\left(\Delta^{c}\right)$. Furthermore $\left(\Delta^{c}\right)^{c}=\Delta$, and it is known that $\Delta$ is a matroid if and only if $\Delta^{c}$ is a matroid ( $\underline{\mathrm{Ox}}$, Theorem 2.1.1]). Actually the matroid $\Delta^{c}$ is known as the dual of $\Delta$.

In order to have a good combinatorial description of $J(\Delta)^{(m)}$ we need a concept that is more general than a vertex cover: For a natural number $k$, a $k$-cover of $\Delta$ is a nonzero function

$$
\alpha:[n] \longrightarrow \mathbb{N}
$$

such that $\sum_{i \in F} \alpha(i) \geq k$ for any $F \in \mathcal{F}(\Delta)$. Of course vertex covers and 1-covers with values on $\{0,1\}$ are the same thing. It is not difficult to see that

$$
J(\Delta)^{(m)}=\left(x_{1}^{\alpha(1)} \cdots x_{n}^{\alpha(n)}: \alpha \text { is an } m \text {-cover of } \Delta\right) .
$$

A $k$-cover $\alpha$ of $\Delta$ is said to be basic if for any nonzero function $\beta:[n] \longrightarrow \mathbb{N}$ with $\beta(i) \leq \alpha(i)$ for any $i \in[n]$, if $\beta$ is a $k$-cover of $\Delta$, then $\beta=\alpha$. Of course, to the basic $m$-covers of $\Delta$ there corresponds a minimal system of generators of $J(\Delta)^{(m)}$.

Now let us consider the multiplicative filtration $\operatorname{Symb}(\Delta)=\left\{J(\Delta)^{(m)}\right\}_{m \in \mathbb{N} \geq 1}$. We can form the Rees algebra of $S$ with respect to the filtration $\mathcal{S} y m b(\Delta)$,

$$
A(\Delta)=S \oplus\left(\bigoplus_{m \geq 1} J(\Delta)^{(m)}\right) .
$$

In [HHT, Theorem 3.2], Herzog, Hibi and Trung proved that $A(\Delta)$ is noetherian. In particular, the associated graded ring of $S$ with respect to $\mathcal{S} y m b(\Delta)$,

$$
G(\Delta)=S / J(\Delta) \oplus\left(\bigoplus_{m \geq 1} J(\Delta)^{(m)} / J(\Delta)^{(m+1)}\right),
$$

and the special fiber,

$$
\bar{A}(\Delta)=A(\Delta) / \mathfrak{m} A(\Delta)=G(\Delta) / \mathfrak{m} G(\Delta),
$$

are noetherian too. The algebra $A(\Delta)$ is known as the vertex cover algebra of $\Delta$, and its properties have been intensively studied in [HHT]. The name comes from the fact that, writing

$$
A(\Delta)=S \oplus\left(\bigoplus_{m \geq 1} J(\Delta)^{(m)} \cdot t^{m}\right) \subseteq S[t]
$$


and denoting by $(A(\Delta))_{m}=J(\Delta)^{(m)} \cdot t^{m}$, it turns out that a (infinite) basis for $A(\Delta)_{m}$ as a $K$-vector space is

$$
\left\{x_{1}^{\alpha(1)} \cdots x_{n}^{\alpha(n)} \cdot t^{m}: \alpha \text { is a } m \text {-cover of } \Delta\right\} .
$$

The algebra $\bar{A}(\Delta)$, instead, is called the algebra of basic covers of $\Delta$, and its properties have been studied by the author with Benedetti and Constantinescu in $\mathrm{BCV}$ ] and with Constantinescu in [CV] for a 1-dimensional simplicial complex $\Delta$. Clearly, the grading defined above on $A(\Delta)$ induces a grading on $\bar{A}(\Delta)$, and it turns out that a basis for $(\bar{A}(\Delta))_{m}, m \geq 1$, as a $K$-vector space is

$$
\left\{x_{1}^{\alpha(1)} \cdots x_{n}^{\alpha(n)} \cdot t^{m}: \alpha \text { is a basic } m \text {-cover of } \Delta\right\} .
$$

Notice that if $\alpha$ is a basic $m$-cover of $\Delta$, then $\alpha(i) \leq m$ for any $i \in[n]$. This implies that $(\bar{A}(\Delta))_{m}$ is a finite $K$-vector space for any $m \in \mathbb{N}$. So we can speak about the Hilbert function of $\bar{A}(\Delta)$, denoted by $\mathrm{HF}_{\bar{A}(\Delta)}$, and from what we said above we have, for $k \geq 1$,

$$
\operatorname{HF}_{\bar{A}(\Delta)}(k)=\mid\{\text { basic } k \text {-covers of } \Delta\} \mid \text {. }
$$

The key to prove Theorem 2.1 is to compute the dimension of $\bar{A}(\Delta)$. So we need a combinatorial description of $\operatorname{dim}(\bar{A}(\Delta))$. Being in general non-standard graded, the algebra $\bar{A}(\Delta)$ could not have a Hilbert polynomial. However, by [HHT, Corollary 2.2] we know that there exists $h \in \mathbb{N}$ such that $\left(J(\Delta)^{(h)}\right)^{m}=J(\Delta)^{(h m)}$ for all $m \geq 1$. It follows that $\bar{A}(\Delta)^{(h)}=\bigoplus_{m \in \mathbb{N}}(\bar{A}(\Delta))_{h m}$ is a standard graded $K$ algebra. Notice that if a set $\left\{f_{1}, \ldots, f_{q}\right\}$ generates $\bar{A}(\Delta)$ as a $K$-algebra, then the set $\left\{f_{1}^{i_{1}} \cdots f_{q}^{i_{q}}: 0 \leq i_{1}, \ldots, i_{q} \leq h-1\right\}$ generates $\bar{A}(\Delta)$ as a $\bar{A}(\Delta)^{(h)}$-module. Thus $\operatorname{dim}(\bar{A}(\Delta))=\operatorname{dim}\left(\bar{A}(\Delta)^{(h)}\right)$. Since $\bar{A}(\Delta)^{(h)}$ has a Hilbert polynomial, we get a useful criterion to compute the dimension of $\bar{A}(\Delta)$. First recall that, for two functions $f, g: \mathbb{N} \rightarrow \mathbb{R}$, the writing $f(k)=O(g(k))$ means that there exists a positive real number $\lambda$ such that $f(k) \leq \lambda \cdot g(k)$ for $k \gg 0$. Similarly, $f(k)=\Omega(g(k))$ if there is a positive real number $\lambda$ such that $f(k) \geq \lambda \cdot g(k)$ for $k \gg 0$.

The criterion for detecting the dimension of $\bar{A}(\Delta)$. If $\operatorname{HF}_{\bar{A}(\Delta)}(k)=O\left(k^{d-1}\right)$, then $\operatorname{dim}(\bar{A}(\Delta)) \leq d$. If $\operatorname{HF}_{\bar{A}(\Delta)}(k)=\Omega\left(k^{d-1}\right)$, then $\operatorname{dim}(\bar{A}(\Delta)) \geq d$.

The following proposition justifies the introduction of $\bar{A}(\Delta)$.

Proposition 2.4. For any simplicial complex $\Delta$ on $[n]$ we have

$$
\operatorname{dim}(\bar{A}(\Delta))=n-\min \left\{\operatorname{depth}\left(S / J(\Delta)^{(m)}\right): m \in \mathbb{N}_{\geq 1}\right\} .
$$

Proof. Consider $G(\Delta)$, the associated graded ring of $S$ with respect to $\mathcal{S} y m b(\Delta)$. Since $G(\Delta)$ is noetherian, it follows by Bruns and Vetter BrVe, Proposition 9.23] that

$$
\min \left\{\operatorname{depth}\left(S / J(\Delta)^{(m)}\right): m \in \mathbb{N}_{\geq 1}\right\}=\operatorname{grade}(\mathfrak{m} G(\Delta)) .
$$

We claim that $G(\Delta)$ is Cohen-Macaulay. In fact the Rees ring of $S$ with respect to the filtration $\mathcal{S} y m b(\Delta)$, namely $A(\Delta)$, is Cohen-Macaulay by [HHT, Theorem 4.2]. Let us denote by $A(\Delta)_{+}=\bigoplus_{m>0} J(\Delta)^{(m)}$ and by $\mathfrak{M}=\mathfrak{m} \oplus A(\Delta)_{+}$the unique bi-graded maximal ideal of $A(\Delta)$. The short exact sequence

$$
0 \longrightarrow A(\Delta)_{+} \longrightarrow A(\Delta) \longrightarrow S \longrightarrow 0
$$


yields the long exact sequence on local cohomology:

$$
\begin{aligned}
\ldots & \rightarrow H_{\mathfrak{M}}^{i}\left(A(\Delta)_{+}\right) \rightarrow H_{\mathfrak{M}}^{i}(A(\Delta)) \rightarrow H_{\mathfrak{M}}^{i}(S) \rightarrow H_{\mathfrak{M}}^{i+1}\left(A(\Delta)_{+}\right) \\
& \rightarrow H_{\mathfrak{M}}^{i+1}(A(\Delta)) \rightarrow \ldots
\end{aligned}
$$

By the independence of the base in computing local cohomology modules we have $H_{\mathfrak{M}}^{i}(S)=H_{\mathfrak{m}}^{i}(S)=0$ for any $i<n$. Furthermore, $H_{\mathfrak{M}}^{i}(A(\Delta))=0$ for any $i \leq n$ since $A(\Delta)$ is a Cohen-Macaulay $(n+1)$-dimensional (see [BH, Theorem 4.5.6]) ring. Thus $H_{\mathfrak{M}}^{i}\left(A(\Delta)_{+}\right)=0$ for any $i \leq n$ by the above long exact sequence. Now let us look at the other short exact sequence,

$$
0 \longrightarrow A(\Delta)_{+}(1) \longrightarrow A(\Delta) \longrightarrow G(\Delta) \longrightarrow 0,
$$

where $A(\Delta)_{+}(1)$ means $A(\Delta)_{+}$with the degrees shifted by 1 , and the corresponding long exact sequence on local cohomology,

$$
\ldots \rightarrow H_{\mathfrak{M}}^{i}\left(A(\Delta)_{+}(1)\right) \rightarrow H_{\mathfrak{M}}^{i}(A(\Delta)) \rightarrow H_{\mathfrak{M}}^{i}(G(\Delta)) \rightarrow H_{\mathfrak{M}}^{i+1}\left(A(\Delta)_{+}(1)\right) \rightarrow \ldots
$$

Because $A(\Delta)_{+}$and $A(\Delta)_{+}(1)$ are isomorphic $A(\Delta)$-modules, $H_{\mathfrak{M}}^{i}\left(A(\Delta)_{+}(1)\right)=0$ for any $i \leq n$. Thus $H_{\mathfrak{M}}^{i}(G(\Delta))=0$ for any $i<n$. Since $G(\Delta)$ is an $n$-dimensional ring (see $\underline{\mathrm{BH}}$, Theorem 4.5.6]), this implies, once again using the independence of the base in computing local cohomology, that $G(\Delta)$ is Cohen-Macaulay.

Since $G(\Delta)$ is Cohen-Macaulay, grade $(\mathfrak{m} G(\Delta))=\operatorname{ht}(\mathfrak{m} G(\Delta))$. So, because $\bar{A}(\Delta)$ $=G(\Delta) / \mathfrak{m} G(\Delta)$, we get

$$
\operatorname{dim}(\bar{A}(\Delta))=\operatorname{dim}(G(\Delta))-\operatorname{ht}(\mathfrak{m} G(\Delta))=n-\operatorname{grade}(\mathfrak{m} G(\Delta)),
$$

and the statement follows.

We are almost ready to show Theorem 2.1. We need just a technical lemma which allows us to construct "many" basic covers.

Lemma 2.5. Let $s \geq-1$ and $d$ be integer numbers such that $s \leq d-3$. For any positive integer $k$ consider the set

$$
\begin{aligned}
A_{k}= & \left\{\left(a_{1}, a_{2}, \ldots, a_{d}, b_{1}, b_{2}, \ldots, b_{d-s-1}\right) \in \mathbb{N}^{2 d-s-1}:\right. \\
& a_{1}+\ldots+a_{d}=k, a_{1}+\ldots+a_{d-s+1}=b_{1}+\ldots+b_{d-s-1}, \\
& \left.a_{1} \geq a_{2} \geq \ldots \geq a_{d}, \quad \text { and } b_{1}, b_{2}, \ldots, b_{d-s-1} \geq a_{2}\right\} .
\end{aligned}
$$

Then $\left|A_{k}\right|=\Omega\left(k^{2 d-s-3}\right)$.

Proof. Let us set

$$
X_{k}=\left\{a_{1} \in \mathbb{N}: \frac{(d+1) k}{d+2} \leq a_{1} \leq \frac{(d+2) k}{d+3}\right\} .
$$

Of course, setting $\lambda_{1}=\frac{1}{(d+2)(d+3)}$, we have $\left|X_{k}\right| \geq \lambda_{1} \cdot k$.

For a fixed $a_{1} \in X_{k}$, set

$$
Y_{k}\left(a_{1}\right)=\left\{\left(a_{2}, \ldots, a_{d}\right): a_{1}+a_{2}+\ldots+a_{d}=k\right\} .
$$

The vectors $\left(a_{2}, \ldots, a_{d}\right) \in Y_{k}\left(a_{1}\right)$ are as many as the integer partitions of $k-a_{1}$ with at most $d-1$ parts. Because $a_{1} \in X_{k}$ these are at least as many as the partitions $\lfloor k /(d+3)\rfloor$ with at most $d-1$ parts. These, in general, are less than all the monomials of degree $\lfloor k /(d+3)\rfloor$ in $d-1$ variables, i.e. $\left(\begin{array}{c}d-2+\lfloor k /(d+3)\rfloor \\ d-2\end{array}\right)$, since a permutation of the variables gives the same partitions but may give different 
monomials. Anyway, since this is the only reason, the number of the possible $\left(a_{2}, \ldots, a_{d}\right)$ is at least

$$
\frac{1}{(d-1) !}\left(\begin{array}{c}
d-2+\lfloor k /(d+3)\rfloor \\
d-2
\end{array}\right) .
$$

So there exists a positive real number $\lambda_{2}$, independent on $a_{1}$, such that $\left|Y_{k}\left(a_{1}\right)\right| \geq$ $\lambda_{2} \cdot k^{d-2}$.

Let $\mathbf{a}=\left(a_{1}, a_{2}, \ldots, a_{d}\right)$ be a vector such that $a_{1} \in X_{k}$ and $\left(a_{2}, \ldots, a_{d}\right) \in Y_{k}\left(a_{1}\right)$. Then set

$$
Z_{k}(\mathbf{a})=\left\{\left(b_{1}, \ldots, b_{d-s-1}\right) \in \mathbb{N}_{\geq a_{2}}^{d-s-1}: b_{1}+\ldots+b_{d-s-1}=a_{1}+\ldots+a_{d-s-1}\right\} .
$$

It is easy to notice that the vectors $\left(b_{1}, \ldots, b_{d-s-1}\right) \in Z_{k}(\mathbf{a})$ are as many as all the monomials of degree $a_{1}+\ldots+a_{d-s-1}-(d-s-1) a_{2}$ in $d-s-1$ variables. Clearly we have

$$
a_{1}+\ldots+a_{d-s-1}-(d-s-1) a_{2} \geq a_{1}-(d-s-1) a_{2},
$$

but $a_{2} \leq k-a_{1} \leq \frac{k}{d+2}$. So we get

$a_{1}+\ldots+a_{d-s-1}-(d-s-1) a_{2} \geq a_{1}-(d-s-1) a_{2} \geq \frac{(d+1) k}{d+2}-\frac{d k}{d+2}=\frac{k}{d+2}$.

So the elements of $Z_{k}(\mathbf{a})$ are at least as many as the monomials of degree $\lfloor k /(d+2)\rfloor$ in $d-s-1$ variables. Therefore there is a positive real number $\lambda_{3}$, not depending on $\mathbf{a}$, such that $\left|Z_{k}(\mathbf{a})\right| \geq \lambda_{3} \cdot k^{d-s-2}$.

Finally, we have that

$$
\begin{aligned}
\left|A_{k}\right| & \geq \sum_{a_{1} \in X_{k}} \sum_{\left(a_{2}, \ldots, a_{d}\right) \in Y_{k}\left(a_{1}\right)}\left|Z_{k}(\mathbf{a})\right| \geq\left(\lambda_{1} \cdot k\right) \cdot\left(\lambda_{2} \cdot k^{d-2}\right) \cdot\left(\lambda_{3} \cdot k^{d-s-2}\right) \\
& =\lambda_{1} \lambda_{2} \lambda_{3} \cdot k^{2 d-s-3} .
\end{aligned}
$$

Now we are ready to prove Theorem 2.1 ,

Proof. By the duality on the matroids it is enough to prove that $S / J(\Delta)^{(m)}$ is Cohen-Macaulay for any $m \in \mathbb{N}_{\geq 1}$ if and only if $\Delta$ is a matroid. Suppose that $\Delta$ is $(d-1)$-dimensional.

The if-part. Let us consider a basic $k$-cover $\alpha$ of $\Delta$. Let $F$ be a facet of $\Delta$ such that $\sum_{j \in F} \alpha(j)=k$ ( $F$ exists because $\alpha$ is basic). Set

$$
A_{F}=\{\alpha(j): j \in F\} .
$$

We claim that for any $i \in[n]$ we have $\alpha(i) \in A_{F}$. In fact, if $i_{0} \in[n]$ does not belong to $F$, then, because $\alpha$ is basic, there exists a facet $G$ of $\Delta$ such that $i_{0} \in G$ and $\sum_{i \in G} \alpha(i)=k$. By the exchange property there exists a vertex $j_{0} \in F$ such that $\left(G \backslash\left\{i_{0}\right\}\right) \cup\left\{j_{0}\right\}$ and $\left(F \backslash\left\{j_{0}\right\}\right) \cup\left\{i_{0}\right\}$ are facets of $\Delta$. But

$$
\sum_{i \in\left(G \backslash\left\{i_{0}\right\}\right) \cup\left\{j_{0}\right\}} \alpha(i) \geq k \Longrightarrow \alpha\left(j_{0}\right) \geq \alpha\left(i_{0}\right)
$$

and

$$
\sum_{j \in\left(F \backslash\left\{j_{0}\right\}\right) \cup\left\{i_{0}\right\}} \alpha(j) \geq k \Longrightarrow \alpha\left(i_{0}\right) \geq \alpha\left(j_{0}\right) .
$$


Hence $\alpha\left(i_{0}\right)=\alpha\left(j_{0}\right) \in A_{F}$. The number of ways to give values on vertices of $F$ such that the sum of the values on the whole $F$ is $k$ are $\left(\begin{array}{c}k+d-1 \\ d-1\end{array}\right)$. This implies that, for $k \geq 1$,

$\operatorname{HF}_{\bar{A}(\Delta)}(k)=\mid\{$ basic $k$-covers of $\Delta\}|\leq| \mathcal{F}(\Delta) \mid \cdot\left(\begin{array}{c}k+d-1 \\ d-1\end{array}\right) \leq\left(\begin{array}{c}n \\ d\end{array}\right) \cdot\left(\begin{array}{c}k+d-1 \\ d-1\end{array}\right)$.

So $\operatorname{HF}_{\bar{A}(\Delta)}(k)=O\left(k^{d-1}\right)$; therefore $\operatorname{dim}(\bar{A}(\Delta)) \leq d$. But $\operatorname{dim}(S / J(\Delta))=n-d$, so by Proposition 2.4

$$
d \geq \operatorname{dim}(\bar{A}(\Delta))=n-\min \left\{\operatorname{depth}\left(S / J(\Delta)^{(m)}\right): m \in \mathbb{N}_{\geq 1}\right\} \geq d,
$$

from which $S / J(\Delta)^{(m)}$ is Cohen-Macaulay for any $m \in \mathbb{N}_{\geq 1}$.

The only if-part. Suppose to the contrary that $\Delta$ is not a matroid. Then there exist two facets $F$ and $G$ of $\Delta$ and a vertex $i \in F$ such that $(F \backslash\{i\}) \cup\{j\}$ is not a facet of $\Delta$ for any $j \in G$. Let $s$ be the greatest integer such that there exists an $s$-dimensional subface $F^{\prime}$ of $F \backslash\{i\}$ such that there is a $(d-s-2)$-dimensional subface of $G$ whose union with $F^{\prime}$ is a facet of $\Delta$. Notice that $s \leq d-3$ and $s$ might be -1 . Let $F_{0} \subseteq F \backslash\{i\}$ be an $s$-dimensional face and $G_{0} \subseteq G$ a $(d-s-2)$ dimensional face satisfying the above conditions. Let $\left(a_{1}, \ldots, a_{d}, b_{1}, \ldots, b_{d-s-1}\right) \in$ $A_{k}$, where $A_{k}$ is the set defined in Lemma 2.5. Set $F=\left\{i_{1}, \ldots, i_{d}\right\}$ with $i_{1}=i$ and $F_{0}=\left\{i_{d-s}, \ldots, i_{d}\right\}$. Also, set $G=\left\{j_{1}, \ldots, j_{d}\right\}$ where $G_{0}=\left\{j_{1}, \ldots, j_{d-s-1}\right\}$. Now we define the following numerical function on $[n]$ :

$$
\alpha^{\prime}(v)= \begin{cases}a_{p} & \text { if } v=i_{p}, \\ b_{q} & \text { if } v=j_{q} \text { and } q<d-s, \\ k & \text { otherwise. }\end{cases}
$$

We claim that $\alpha^{\prime}$ is a $k$-cover, not necessarily basic. By the definition of $\alpha^{\prime}$ we have to check that for any facet $H$ of $\Delta$ contained in $F \cup G_{0}$ we have the inequality $\sum_{h \in H} \alpha^{\prime}(h) \geq k$. If $i \notin H$, then $G_{0} \subset H$ by the maximality of $s$. But then we have

$$
\sum_{h \in H} \alpha^{\prime}(h)=\sum_{h \in G_{0}} \alpha^{\prime}(h)+\sum_{h \in H \backslash G_{0}} \alpha^{\prime}(h) \geq \sum_{h \in G_{0}} \alpha^{\prime}(h)+\sum_{h \in F_{0}} \alpha^{\prime}(h)=k .
$$

If $i \in H$, then we have

$$
\begin{aligned}
\sum_{h \in H} \alpha^{\prime}(h) & =a_{1}+\sum_{h \in H \cap(F \backslash\{i\})} \alpha^{\prime}(h)+\sum_{h \in H \backslash F} \alpha^{\prime}(h) \\
& \geq a_{1}+\sum_{h \in H \cap(F \backslash\{i\})} \alpha^{\prime}(h)+|H \backslash F| \cdot a_{2} \\
& \geq a_{1}+\ldots+a_{d}=k .
\end{aligned}
$$

Reducing the values of $\alpha^{\prime}$ where possible, we can make $\alpha^{\prime}$ in a basic $k$-cover $\alpha$. However we cannot reduce the values at the vertices of $F \cup G_{0}$ because of the equalities

$$
\sum_{h \in F} \alpha^{\prime}(h)=k \quad \text { and } \quad \sum_{h \in F_{0} \cup G_{0}} \alpha^{\prime}(h)=k .
$$

Thus the basic $k$-covers of $\mathcal{F}(\Delta)$ are at least as many as the cardinality of $A_{k}$. So by Lemma 2.5 there exists a positive real number $\lambda$ such that for $k \gg 0$ we have

$$
\operatorname{HF}_{\bar{A}(\Delta)}(k)=\mid\{\text { basic } k \text {-covers of } \Delta\} \mid \geq \lambda \cdot k^{2 d-s-3} \geq \lambda \cdot k^{d} .
$$

So $\operatorname{HF}_{\bar{A}(\Delta)}(k)=\Omega\left(k^{d}\right)$; therefore $\operatorname{dim}(\bar{A}(\Delta)) \geq d+1$. Using Proposition 2.4 we have that

$$
\min \left\{\operatorname{depth}\left(S / J(\Delta)^{(m)}\right): m \in \mathbb{N}_{\geq 1}\right\} \leq n-d-1,
$$


which contradicts the hypothesis that $S / J(\Delta)^{(m)}$ is Cohen-Macaulay for any $m \in$ $\mathbb{N}_{\geq 1}$.

We end the paper by stating two corollaries of Theorem 2.1. First we recall that the multiplicity of a standard graded $K$-algebra $R$, denoted by $e(R)$, is the leading coefficient of the Hilbert polynomial times $(\operatorname{dim}(R)-1)$ !. Geometrically, let $\operatorname{Proj} R \subseteq \mathbb{P}^{N}$; i.e. $R=K\left[X_{0}, \ldots, X_{N}\right] / J$ for a homogeneous ideal $J$. The multiplicity $e(R)$ counts the number of distinct points of $\operatorname{Proj} R \cap H$, where $H$ is a generic linear subspace of $\mathbb{P}^{N}$ of dimension $N-\operatorname{dim}(\operatorname{Proj} R)$.

Corollary 2.6. A simplicial complex $\Delta$ is a $(d-1)$-dimensional matroid if and only if

$$
\operatorname{dim}(\bar{A}(\Delta))=\operatorname{dim}(K[\Delta])=d .
$$

Moreover, if $\Delta$ is a matroid, then

$$
\operatorname{HF}_{\bar{A}(\Delta)}(k) \leq \frac{e(K[\Delta])}{(\operatorname{dim}(\bar{A}(\Delta))-1) !} k^{\operatorname{dim}(\bar{A}(\Delta))-1}+O\left(k^{\operatorname{dim}(\bar{A}(\Delta))-2}\right) .
$$

Proof. The first fact follows from putting together Theorem 2.1 and Proposition 2.4. For the second fact, we have to recall that during the proof of Theorem 2.1. we showed that for a $(d-1)$-dimensional matroid $\Delta$ we have the inequality

$$
\operatorname{HF}_{\bar{A}(\Delta)}(k) \leq|\mathcal{F}(\Delta)| \cdot\left(\begin{array}{c}
k+d-1 \\
d-1
\end{array}\right)
$$

It is well known that if $\Delta$ is a pure simplicial complex, then $|\mathcal{F}(\Delta)|=e(K[\Delta]$ ) (for instance see [BH, Corollary 5.1.9]), so we get the conclusion.

Example 2.7. If $\Delta$ is not a matroid the inequality of Corollary 2.6 may not be true. For instance, take $\Delta=C_{10}$, the decagon (thus it is a 1-dimensional simplicial complex). Since $C_{10}$ is a bipartite graph, $\bar{A}\left(C_{10}\right)$ is a standard graded $K$-algebra by [HHT, Theorem 5.1]. In particular it admits a Hilbert polynomial, and for $k \gg 0$ we have

$$
\operatorname{HF}_{\bar{A}\left(C_{10}\right)}(k)=\frac{e\left(\bar{A}\left(C_{10}\right)\right)}{\left(\operatorname{dim}\left(\bar{A}\left(C_{10}\right)\right)-1\right) !} k^{\operatorname{dim}\left(\bar{A}\left(C_{10}\right)\right)-1}+O\left(k^{\operatorname{dim}\left(\bar{A}\left(C_{10}\right)\right)-2}\right) .
$$

In [CV] it is proved that for any bipartite graph $G$ the algebra $\bar{A}(G)$ is a homogeneous algebra with straightening law on a poset described in terms of the minimal vertex covers of $G$. So the multiplicity of $\bar{A}(G)$ can be easily read from the above poset. In our case it is easy to check that $e\left(\bar{A}\left(C_{10}\right)\right)=20$, whereas $e\left(K\left[C_{10}\right]\right)=10$.

Let us introduce the last result of the paper. An ideal $I$ of a ring $R$ is a settheoretic complete intersection if there exist $f_{1}, \ldots, f_{h} \in R$, where $h=\operatorname{ht}(I)$, such that $\sqrt{\left(f_{1}, \ldots, f_{h}\right)}=\sqrt{I}$. The importance of this notion comes from algebraic geometry, since if $I$ is a set-theoretic complete intersection, then the variety $\mathcal{V}(I) \subseteq \operatorname{Spec}(R)$ can be defined set-theoretically by "cutting" the "right" number of hypersurfaces of $\operatorname{Spec}(R)$. A necessary, in general not sufficient, condition for $I$ to be a set-theoretic complete intersection is that the cohomological dimension of it, $\operatorname{cd}(R, I)=\max \left\{i: H_{I}^{i}(R) \neq 0\right\}$, is $h$. By a result of Lyubeznik in Ly it turns out that $\operatorname{cd}\left(S, I_{\Delta}\right)=n-\operatorname{depth}(K[\Delta])$, so if $I_{\Delta}$ is a set-theoretic complete intersection, $K[\Delta]$ will be Cohen-Macaulay. 
Remark 2.8. In general if $K[\Delta]$ is Cohen-Maculay, then $I_{\Delta}$ might not be a settheoretic complete intersection. For instance, if $\Delta$ is the triangulation of the real projective plane with 6 vertices described in $[\mathrm{BH}$, p. 236], then $K[\Delta]$ is CohenMacaulay whenever $\operatorname{char}(K) \neq 2$. However, for any characteristic of $K, I_{\Delta}$ need at least (actually exactly) four polynomials of $K\left[x_{1}, \ldots, x_{6}\right]$ to be defined up to radical (see the paper of Yan [Ya, p. 317, Example 2]), but ht $\left(I_{\Delta}\right)=3$.

Corollary 2.9. Let $K$ be an infinite field. For any matroid $\Delta$, the ideal $I_{\Delta} S_{\mathfrak{m}}$ is a set-theoretic complete intersection in $S_{\mathfrak{m}}$.

Proof. By the duality on matroids it is enough to prove that $J(\Delta) S_{\mathfrak{m}}$ is a settheoretic complete intersection. For $h \gg 0$ it follows by [HHT, Corollary 2.2] that the $h$ th Veronese of $\bar{A}(\Delta)$,

$$
\bar{A}(\Delta)^{(h)}=\bigoplus_{m \geq 0} \bar{A}(\Delta)_{h m}
$$

is standard graded. Therefore $\bar{A}(\Delta)^{(h)}$ is the ordinary fiber cone of $J(\Delta)^{(h)}$. Moreover $\bar{A}(\Delta)$ is finite as a $\bar{A}(\Delta)^{(h)}$-module. So the dimensions of $\bar{A}(\Delta)$ and of $\bar{A}(\Delta)^{(h)}$ are the same. Therefore, using Theorem 2.1 and Proposition 2.4, we get

$$
\operatorname{ht}\left(J(\Delta) S_{\mathfrak{m}}\right)=\operatorname{ht}(J(\Delta))=\operatorname{dim} \bar{A}(\Delta)^{(h)}=\ell\left(J(\Delta)^{(h)}\right)=\ell\left(\left(J(\Delta) S_{\mathfrak{m}}\right)^{(h)}\right),
$$

where $\ell(\cdot)$ is the analytic spread of an ideal, i.e. the Krull dimension of its ordinary fiber cone. From a result by Northcott and Rees in [NR, p. 151], since $K$ is infinite, it follows that the analytic spread of $\left(J(\Delta) S_{\mathfrak{m}}\right)^{(h)}$ is the cardinality of a set of minimal generators of a minimal reduction of $\left(J(\Delta) S_{\mathfrak{m}}\right)^{(h)}$. Clearly the radical of such a reduction is the same as the radical of $\left(J(\Delta) S_{\mathfrak{m}}\right)^{(h)}$, i.e. $J(\Delta) S_{\mathfrak{m}}$, so we get the statement.

Remark 2.10. Notice that a reduction of $I S_{\mathfrak{m}}$, where $I$ is a homogeneous ideal of $S$, might not provide a reduction of $I$. So localizing at the maximal irrelevant ideal is a crucial assumption of Corollary 2.9. It would be interesting to know whether $I_{\Delta}$ is a set-theoretic complete intersection in $S$ whenever $\Delta$ is a matroid.

\section{ACKNOWLEDGEMENTS}

The author would like to thank Margherita Barile for valuable information about Corollary 2.9. Aldo Conca for carefully reading the paper, and Le Dinh Nam for useful conversations about the topic of the work.

\section{REFERENCES}

[BCV] B. Benedetti, A. Constantinescu, M. Varbaro, Dimension, depth and zero-divisors of the algebra of basic $k$-covers of a graph, Le Matematiche LXIII, n. II, pp. 117-156, 2008. MR2531656 (2010c:13002)

[BH] W. Bruns, J. Herzog, Cohen-Macaulay rings, Cambridge Studies in Advanced Mathematics, 1993. MR.1251956 (95h:13020)

[BrVe] W. Bruns, U. Vetter, Determinantal rings, Lecture Notes in Mathematics, 1327, SpringerVerlag, 1988. MR 953963(89i:13001)

[CV] A. Constantinescu, M. Varbaro, Koszulness, Krull dimension and other properties of graph-related algebra, available online at arXiv:1004.4980v1, 2010.

[HHT] J. Herzog, T. Hibi, N. V. Trung, Symbolic powers of monomial ideals and vertex cover algebras, Adv. in Math. 210, pp. 304-322, 2007. MR2298826 (2007m:13005) 
[Ly] G. Lyubeznik, On the local cohomology modules $H_{\mathfrak{U}}^{i}(R)$ for ideals $\mathfrak{U}$ generated by monomials in an R-sequence, "Complete Intersections", Lect. Notes in Math., 1092, pp. 214-220, Springer, 1984. MR775884 (86f:14002)

[MS] E. Miller, B. Sturmfels, Combinatorial commutative algebra, Graduate Texts in Mathematics, 227, Springer-Verlag, 2005. MR2110098 (2006d:13001)

[MT] N. C. Minh, N. V. Trung, Cohen-Macaulayness of powers of monomial ideals and symbolic powers of Stanley-Reisner ideals, available online at arXiv:1003.2152v1, 2010.

[NR] D.G. Northcott, D. Rees, Reduction of ideals in local rings, Proc. Cambridge Philos. Soc. 50, pp. 145-158, 1954. MR0059889 (15:596a)

[Ox] J. G. Oxley, Matroid theory, Oxford University Press, 1992. MR.1207587 (94d:05033)

[St] R. P. Stanley, Combinatorics and commutative algebra, Progress in Mathematics, 41, Birkhäuser Boston, 1996. MR 1453579 (98h:05001)

[TY] N. Terai, K. Yoshida, Locally complete intersection Stanley-Reisner ideals, Illinois J. Math. 53, pp. 413-429, 2009. MR2594636

[We] D. J. A. Welsh, Matroid Theory, Academic Press, London, 1976. MR0427112 (55:148)

[Ya] Z. Yan, An étale analog of the Goresky-Macpherson formula for subspace arrangements, J. Pure and App. Alg. 146, pp. 305-318, 2000. MR1742346 (2000k:14041)

Dipartimento di Matematica, Universitì degli Studi di Genova, Via Dodrcaneso 35, 16145, Genova, Italy

E-mail address: varbaro@dima.unige.it 